\title{
Spatial-phase-modulation-based study of polyvinyl-alcohol/acrylamide photopolymers in the low spatial frequency range
}

\author{
Sergi Gallego, ${ }^{1, \star}$ André Márquez, ${ }^{1}$ David Méndez, ${ }^{1}$ Stephan Marini, ${ }^{1}$ \\ Augusto Beléndez, ${ }^{1}$ and Inmaculada Pascual ${ }^{2}$ \\ ${ }^{1}$ Departamento Física, Ingeniería de Sistemas y Teoría de la Señal, Universidad de Alicante, \\ 03690 San Vicente del Raspeig, Alicante, Spain \\ ${ }^{2}$ Departamento de Óptica, Farmacología y Anatomía, Universidad de Alicante, \\ 03690 San Vicente del Raspeig, Alicante, Spain \\ *Corresponding author: sergi.gallego @ua.es
}

Received 21 January 2009; revised 22 June 2009; accepted 3 July 2009; posted 13 July 2009 (Doc. ID 106595); published 23 July 2009

\begin{abstract}
Photopolymers are appealing materials for the fabrication of diffractive optical elements (DOEs). We evaluate the possibilities of polyvinyl-alcohol/acrylamide-based photopolymers to store diffractive elements with low spatial frequencies. We record gratings with different spatial frequencies in the material and analyze the material behavior measuring the transmitted and the reflected orders as a function of exposition. We study two different compositions for the photopolymer, with and without a cross-linker. The values of diffraction efficiency achieved for both compositions make the material suitable to record DOEs with long spatial periods. Assuming a Fermi-Dirac-function-based profile, we fitted the diffracted intensities (up to the eighth order) to obtain the phase profile of the recorded gratings. This analysis shows that it is possible to achieve a phase shift larger than $2 \pi$ rad with steep edges in the periodic phase profile. In the case of the measurements in reflection, we have obtained information dealing with the surface profile, which show that it has a smooth shape with an extremely large phase-modulation depth. (C) 2009 Optical Society of America
\end{abstract}

OCIS codes: $\quad 160.4670,160.5470$.

\section{Introduction}

Photopolymers have many different applications in optics due to their good properties as an optical recording medium. Different spatial frequency ranges can be recorded in standard photopolymers depending on each particular application. In polyvinyl alcohol/acrylamide (PVA/AA) photopolymers, there exists a limitation to store high spatial frequencies (up to 2500 lines $/ \mathrm{mm}$ ). This cutoff spatial frequency is due to the finite size of polymer chains [1,2]. To record this range of high spatial frequencies,

0003-6935/09/224403-11\$15.00/0

(C) 2009 Optical Society of America holographic techniques are required. In this area the photopolymers have been widely studied, in particular, to optimize the new holographic recording disks [3-6]. On the other hand, low spatial frequencies are required in the fabrication of diffractive elements [7-11] and to fabricate waveguide elements [12]. These elements can be generated due to two important properties of photopolymers: the variations in refractive index and thickness during exposure [13-18].

The fidelity of PVA/AA-based photopolymers to record high spatial frequencies has been demonstrated in previous works $[19,20]$. To store spatial frequencies of up to 500 lines $/ \mathrm{mm}$, the species diffusion contributes to record the sinusoidal profile. In this paper 
we evaluate the properties of PVA/AA-based photopolymers to be used as diffractive recording media in the range of low spatial frequencies (let us say less than 100 lines $/ \mathrm{mm}$ ). In this range of spatial frequencies, the species diffusion is much less important in diffractive image formation. Furthermore, in the case of low spatial frequencies, we can also obtain important information about the material properties [21-23]. To record the diffractive elements onto the material, it becomes practical to use a spatial light modulator $[23,24]$. The liquid-crystal display (LCD) used permits us to modify the properties (phase and intensity) of the incident wavefront [25]. In particular, the intensity of the transmittance modulation is used for the present studies. We can record different diffractive optics elements systematically. In this work we focus our attention on the material behavior when different spatial frequencies are recorded. We analyze the changes in the shape of the phase gratings obtained during the recording process. To obtain a deeper insight into the profile of the generated gratings, and especially into the sharpness of the edges and the phase-modulation depth, we have obtained numerically the diffraction-efficiency values for the different orders using a Fermi-Dirac-function-based profile [26]. By fitting the numerical results with the experimental ones, we can sketch the grating profile. We can also evaluate the importance of the species diffusion for the shape of the edges. In particular, we study the material behavior for the following spatial grating periods: $0.168,0.336$, and $0.672 \mathrm{~mm}$ (i.e., the spatial frequencies are between less than 2 lines $/ \mathrm{mm}$ and 6 lines $/ \mathrm{mm}$ ). These long periods are in the range of the diffusion length for the monomer.

\section{Experimental}

\section{A. Fitted Profile}

The Fermi-Dirac-based profile is a better option than the super-Gaussian-based profile used in previous works [26] for two main reasons. The parameters in the Fermi-Dirac function are continuous whereas, in the super-Gaussian function, the parameter describing the sharpness of the edges is discrete, thus there is no gradual transition between the fitted profiles. Furthermore, the Fermi-Dirac function provides a very good fit to the cosine function, which may be considered as a reasonable limiting case when edges become highly smoothed by diffusion. The mathematical expression for the amplitude transmission $t(x)$ of a transparent phase element showing a Fermi-Dirac phase profile $\varphi(x)$ can be written as

$$
t(x)=\exp (i \varphi(x))
$$

$$
\varphi(x)=\varphi_{0}\left(1+\exp \left(\alpha \cdot\left(\frac{|x|}{\Omega}-1\right)\right)\right)^{-1}
$$

where $\varphi_{0}$ is the asymptotic phase-modulation depth (for an $x$ interval of $(-\infty,+\infty)$ ), $\Omega$ controls the symmetry (or duty cycle) of the profile, and $\alpha$ is the parameter characterizing the function shape and sharpness of the edges. The function is limited to an $x$ interval of $(-1,1)$, and is repeated periodically. We consider symmetric gratings that, for this $x$ interval, correspond to the value $\Omega=0.5$. We rescale the phase values obtained in the $x$-interval $(-1,1)$ so that the phase-modulation depth in this finite interval equals the asymptotic phase-modulation depth. In Fig. 1(a) we show the normalized profile for the Fermi-Dirac function in the $x$ interval $(1,-1)$, for $\Omega=$ 0.5 (symmetric profile), and for different values of $\alpha$. We see that, for $\alpha=1$, the profile corresponds to a triangle function; for $\alpha=3.3$, it is close to a cosine function; and with an increase in $\alpha$, the profile approaches a rectangle function. In Fig. 1(b) we compare the profile given by a super-Gaussian and a Fermi-Dirac function to a cosine function. We

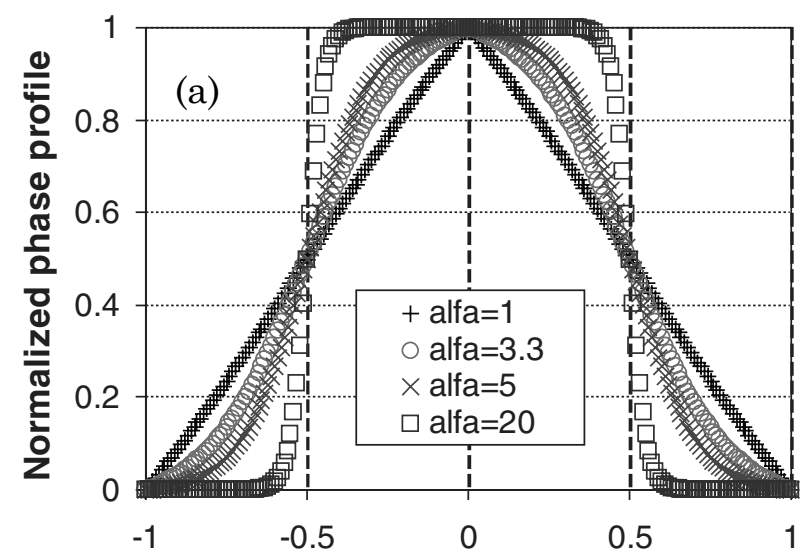

Normalized $\mathrm{x}$ coordinate

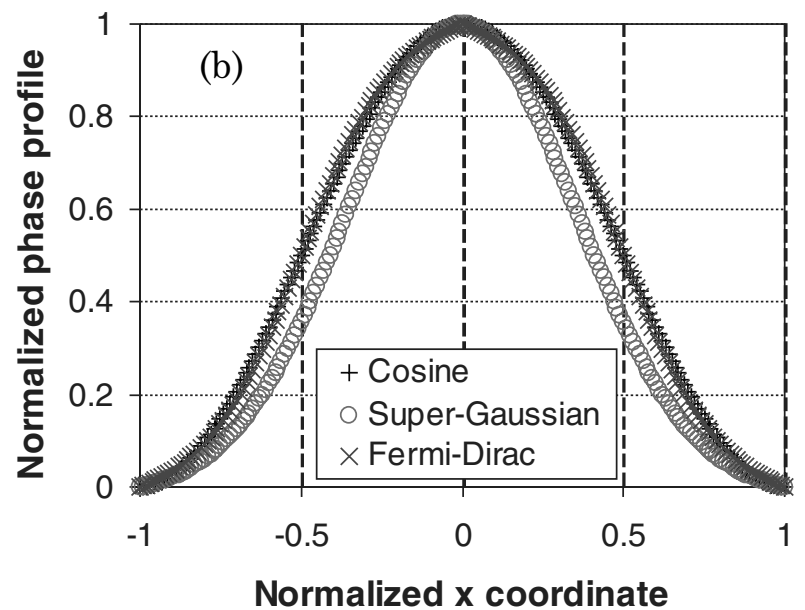

Fig. 1. Normalized phase profiles. (a) Fermi-Dirac profiles for different values of $\alpha$ and for $\Omega=0.5$ (symmetric gratings). (b) Super-Gaussian and Fermi-Dirac best fits to a cosine function, where we show that the Fermi-Dirac function (with $\alpha=3.3$ ) provides a very good approximation. 
see that the Fermi-Dirac function with $\alpha=3.3$ is a good approximation to the cosine. The best superGaussian fit is given by the Gaussian function itself [26], which is clearly a worse approximation.

We have compared the diffraction efficiencies calculated numerically using the Fermi-Dirac model with the experimental values for the first nine orders (orders $0,1, \ldots$, and 8). In Subsection 3.C we will show the results that have allowed obtaining the profile, both in transmission and in reflection, for the phase gratings stored in the photopolymer material.

\section{B. Experimental Setup}

The experimental setup used is shown in Fig. 2. We use an expanded, collimated recording beam provided by a solid-state Verdi laser (Nd:YVO4) with a wavelength of $532 \mathrm{~nm}$ (at this wavelength the dye, yellowish Eosin, exhibits maximum absorption). The exposure intensity is $0.5 \mathrm{~mW} / \mathrm{cm}^{2}$. The periodic pattern is introduced by a LCD, a Sony LCD model LCX012BL, extracted from a video projector, Sony VPL-V500. We use the electronics of the video projector to send the voltage to the pixels of the LCD. The LCD is used in the amplitude-mostly modulation regime by proper orientation of the external polarizers (P) [23], then the pattern is imaged onto the material with an increased spatial frequency (a demagnifying factor of 2). The use of the LCD allows us to change the period of the grating recorded in the photopolymer without moving any mechanical part of the setup. To analyze in real time the variation in efficiency of the different diffraction orders, we use an unexpanded beam of a He-Ne laser $(633 \mathrm{~nm})$ inciding at a small angle with respect to the normal axis of the recording material. A diaphragm (Stop2) is placed in the focal plane of the relay lens so as to eliminate the diffraction orders produced by the pixelation of the LCD. In this work the patterns sent to the LCD correspond to binary gratings with different periods. It can be expected that the final pattern imaged onto the recording material will be partially low filtered due to the finite aperture of the imaging system and especially due to the filtering produced by Stop2. However, the periods of the gratings addressed onto the LCD have been kept long enough so that we can still consider that the image on the recording plane is rather close to a binary grating.

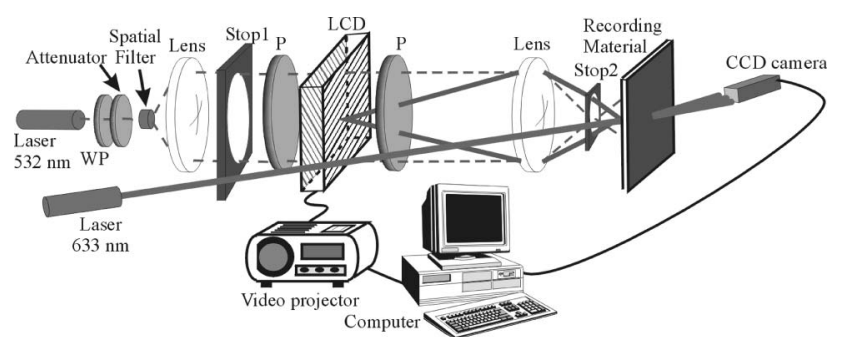

Fig. 2. Experimental setup (transmission measurements). The recording material is exposed to the green laser beam $(\lambda=532 \mathrm{~nm})$ and diffracted efficiencies are generated with the red beam $(\lambda=633 \mathrm{~nm})$. P correspond to the polarizers and WP to the wave plate.
In the results in Subsection 3.C it will become clear that, at least for low exposition times in the recording process, this binary profile is basically maintained when registered into a phase profile.

To obtain the Fourier transform of the pattern, to measure the diffracted intensities, we place a lens after the grating (recorded in the photopolymer). The Fourier plane is captured using a CCD camera at the focal plane of the lens. We use the CCD camera model pco.1600 from pco.imaging. This is a high dynamic 14 bit cooled CCD camera system with a resolution of $1600 \times 1200$ pixels, and a pixel size of $7.4 \mu \mathrm{m} \times 7.4 \mu \mathrm{m}$. The high dynamic range, together with the high number of pixels, allows using this camera as a radiometer where the intensity directed to the various diffraction orders (in our case nine orders) can be measured in parallel. As an example, we show in Fig. 3 the different orders captured by the CCD camera for a grating with a period of $0.672 \mathrm{~mm}$. We have experimentally estimated the error of the diffraction efficiencies obtained using the camera and it turns out to be around $0.5 \%$.

The photopolymers used to store the gratings are similar to the ones used in previous works [22]. The PVA/AA formulations contain a dye (yellowish Eosin), a cosensitizer, which is triethanolamine (TEA), one or two monomers [AA with butyl methacryate (BMA) or without it] and a polymer as a binder (PVA). We have analyzed the grating phase modulation for two different cases: with and without BMA.

\section{Results and Discussion}

We have studied the viability of the photopolymer to record DOEs with spatial periods between 0.168 and $0.672 \mathrm{~mm}$ for two different material compositions with and without a cross-linker. The presence of the cross-linker permits us to achieve higher phase modulations in the objects recorded [22]. On the other hand, we have recently demonstrated that shrinkage increases when a cross-linker is used at the zero spatial frequency limit [22]. In addition, in order to compare the volume changes for the different spatial frequencies, we have measured the main reflected orders to obtain additional information of the surface changes during the recording process.

\section{A. Transmission Orders}

As we already remarked in the introduction, phase grating can be stored in photopolymers due to two phenomena: the volume changes in the surface and the refractive index variation in the polymerization

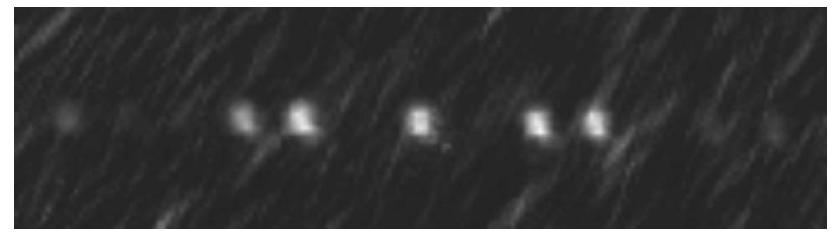

Fig. 3. Image captured by CCD camera when a grating with $0.672 \mathrm{~mm}$ of period is recorded. 
zones. In this section we analyze the two effects together, studying the transmitted orders.

\section{Material Without Cross-Linker}

Recent works based on interferometric optical surface profilometers [17] have demonstrated the existence of a thickness increase in the illuminated areas of some photopolymers for spatial frequencies of 10 lines $/ \mathrm{mm}$. This swelling is due to the incorporation of monomer molecules from the dark zones by diffusion processes. On the other hand, shrinkage of around $4 \%$ has been measured at the zero spatial frequency limit [22] where diffusion does not take place. In this sense, considering the highest values of monomer diffusivity in PVA/AA materials $\left(D=5 \times 10^{-7} \mathrm{~cm}^{2} / \mathrm{s}[23]\right)$ and that exposition stops after $500 \mathrm{~s}$, we can estimate the average molecule diffusion length as follows:

$$
x=\sqrt{D t}=0.05 \mathrm{~mm} .
$$

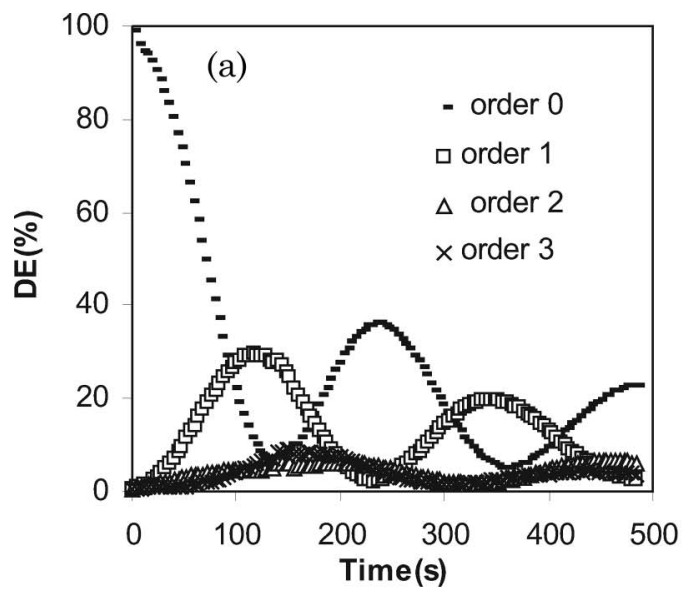

Taking into account that the highest spatial frequency used in our work is around 6 lines $/ \mathrm{mm}$, with a grating period of $0.168 \mathrm{~mm}$, this value indicates that diffusion should become important in the grating formation (the molecules can move almost a third of the spatial period). In this section we have quantified the variations in the material response for a range of spatial periods from 0.168 to $0.672 \mathrm{~mm}$. At this range of spatial frequencies diffusion starts to play an important role in the phase-image formation, making our study fundamental. For spatial periods longer than 0.672 we can expect a similar behavior to the one observed at the zero spatial frequency limit.

In Fig. 4 we present the diffractive efficiency for the first four orders for different grating periods $(0.168,0.336$, and $0.672 \mathrm{~mm})$ as a function of the exposure time. The most significant fact sketched in the figure is the instability of the diffraction efficiencies after long exposure times. For the $0.16 \mathrm{~mm}$ case, the stabilization of diffraction efficiencies cannot be observed after $500 \mathrm{~s}$ (corresponding to about $250 \mathrm{~mJ} / \mathrm{cm}^{2}$ ). This fact constitutes an

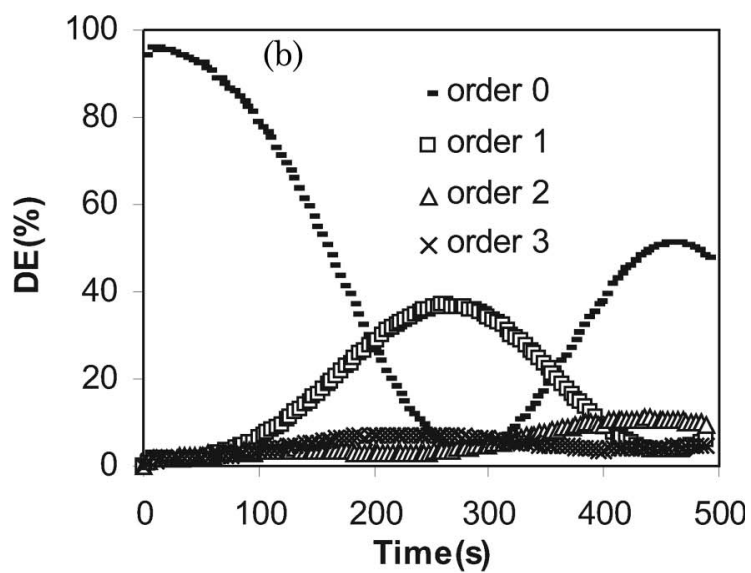

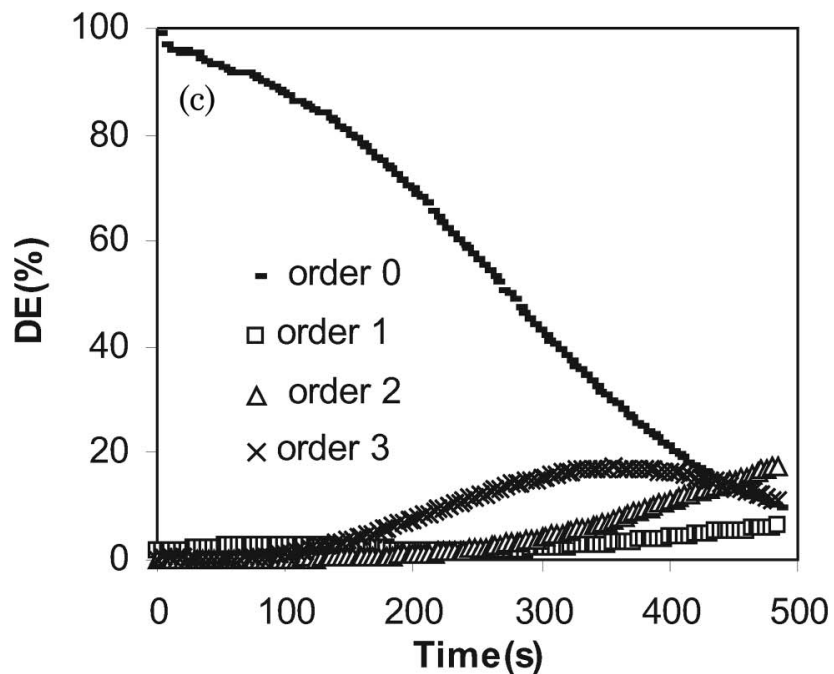

Fig. 4. Diffraction efficiencies of the first four orders for different gratings periods $[0.168,0.336$, and $0.672 \mathrm{~mm}$ correspond to (a), (b), and (c), respectively] as a function of the exposition time ( $105 \mu \mathrm{m}$ thick, without cross-linker, in transmission). 
important difference in comparison with the zero frequency limit case [22], where, after $200 \mathrm{~s}$ (about $80 \mathrm{~mJ} / \mathrm{cm}^{2}$ ), the phase shift becomes constant. The phenomenon can be explained by taking into account the influence of the species diffusion in the refractive index distribution and in the layer profile [22]. In particular, there exists a mixture of three different effects: the changes in the refractive index and the shrinkage and the swelling of the material. The changes in the refractive index of the material are due to polymerization and the existence of holes [22]. Shrinkage is due to the smaller volume of poly-acrylamide chains compared to the volume of the same amount of monomer distributed individually. Swelling can be related to species diffusion from the dark layer areas to the bright ones. The swelling is maximum for periods of around $0.1 \mathrm{~mm}$ [17], although it depends on the periods of the gratings recorded [17]. We observe a smaller material sensitivity (defined as the necessary energy to achieve the first minimum of diffraction efficiency of the zero order) as the period increases, a fact that indicates a minor change in the material surface due to the decreasing effect of diffusion. In particular, for the longer period $(0.672 \mathrm{~mm})$, both the sensitivity and the diffraction efficiency for the first order are very low, indicating that, for much longer periods, the diffusion is minimum. Finally, it is important to note the high values of diffraction efficiencies achieved for thin gratings, larger than $20 \%$ in the first two cases. This parameter is fundamental to using the material to record DOEs. In principle, generic DOEs may be thought of as a composition of different spatial frequencies. In this sense, it would be desirable to obtain similar diffraction efficiencies and sensitivities for the various frequencies. From the results in Fig. 4 we see that the values of these parameters are different for the various frequencies. In this case, a trade-off value for the time exposure providing high diffraction efficiency across the spatial frequency range of interest should be considered when recording a generic DOE.

\section{Cross-Linker Influence}

The importance of cross-linker monomers in photopolymer layers has been studied in many works. It is well known that the introduction of these chemical components in the material composition produces higher values of the polymerization velocities and the shrinkage. To complete our study about the viability of PVA/AA-based material to record structures with very long periods, we have carried out the same experiments using a composition without BMA as cross-linker. In Fig. 5, we present the diffraction efficiency of the four more significant orders for the same spatial frequencies as in Subsection 3.A. Although we observe a similar behavior to the one in the case with a cross-linker, we should not forget to mention that, in this case, energetic sensitivity is clearly higher. This fact can be deduced if Figs. $\underline{4}$ and
5 are compared. The results for very long periods are very similar [Fig. 5(c)]. The maximum of diffraction efficiency is again larger than 20\% in the first two cases.

\section{B. Reflection Orders}

As we have mentioned in Section 1 , when a photopolymer is illuminated, there are refractive index changes and also material volume variations. To have an approximate idea of these two effects, we have analyzed the efficiency of the reflected diffracted orders, which will specifically show material volume variations. The diffraction efficiencies of the first four diffracted orders as a function of the exposure time are depicted in Fig. 6 for the spatial periods of 0.168 and $0.336 \mathrm{~mm}$ in the non-cross-linker case [Figs. 6(a) and 6(b), respectively). In all the gratings we obtain a diffraction efficiency for the first order higher than $20 \%$, interesting for DOEs, for an energetic sensitivity lower than $25 \mathrm{~mJ} / \mathrm{cm}^{2}$. We have chosen not to depict the figure for period $0.672 \mathrm{~mm}$ because the evolution is very similar to Fig. 6(b) (for a period of $0.336 \mathrm{~mm}$ ). On the other hand, we observe clear differences in the diffraction efficiency evolution between Figs. $\underline{6(\mathrm{a})}$ and $\underline{6(\mathrm{~b})}$ in the first $200 \mathrm{~s}$

At this point, it is important to remark that, for all different spatial frequencies, the efficiencies of each order decrease to values lower than a $10 \%$ after $200 \mathrm{~s}$; this fact did not occur in the case of the transmitted orders, as can be seen in Subsection 3.A. Experimentally, we obtain that, as the time increases the total sum of the intensity diffracted to the first 17 orders $(0, \pm 1, \ldots, \pm 8)$ becomes close to zero. This is an unexpected result since, intuitively, it seems that a significant portion of the energy should be contained in the first diffracted orders. To gain some insight into the origin of these results, we have used the Fermi-Dirac model presented in Subsection 2.A to calculate the sum of the intensity directed to the first 17 orders as a function of the phase depth of the grating and for different values of the $\alpha$ parameter. In Fig. 7 we have represented these results. As can be seen for values of $\alpha$ lower than 5 , the first eight diffraction orders are very weak when the phase depth is large. Therefore, this effect indicates that large values of the phase modulation are obtained and that they correspond to smoothed profiles [see Fig. 1(a) for the profile corresponding to $\alpha=5$ ). In [22] we demonstrated experimentally than the phase depth can be higher than $10 \pi \mathrm{rad}$. In general, thin gratings with such large modulation depths are not produced, since typically the range of interest is about $2 \pi \mathrm{rad}$, i.e., very far from the more than $10 \pi \mathrm{rad}$ achievable in reflection with the photopolymer under analysis. To our knowledge, this theoretical and experimental analysis for thin phase gratings with very large phase modulations has not been done until now.

In Fig. 8, we show the same characteristic curves for a photopolymer with BMA as a cross-linker (for a 

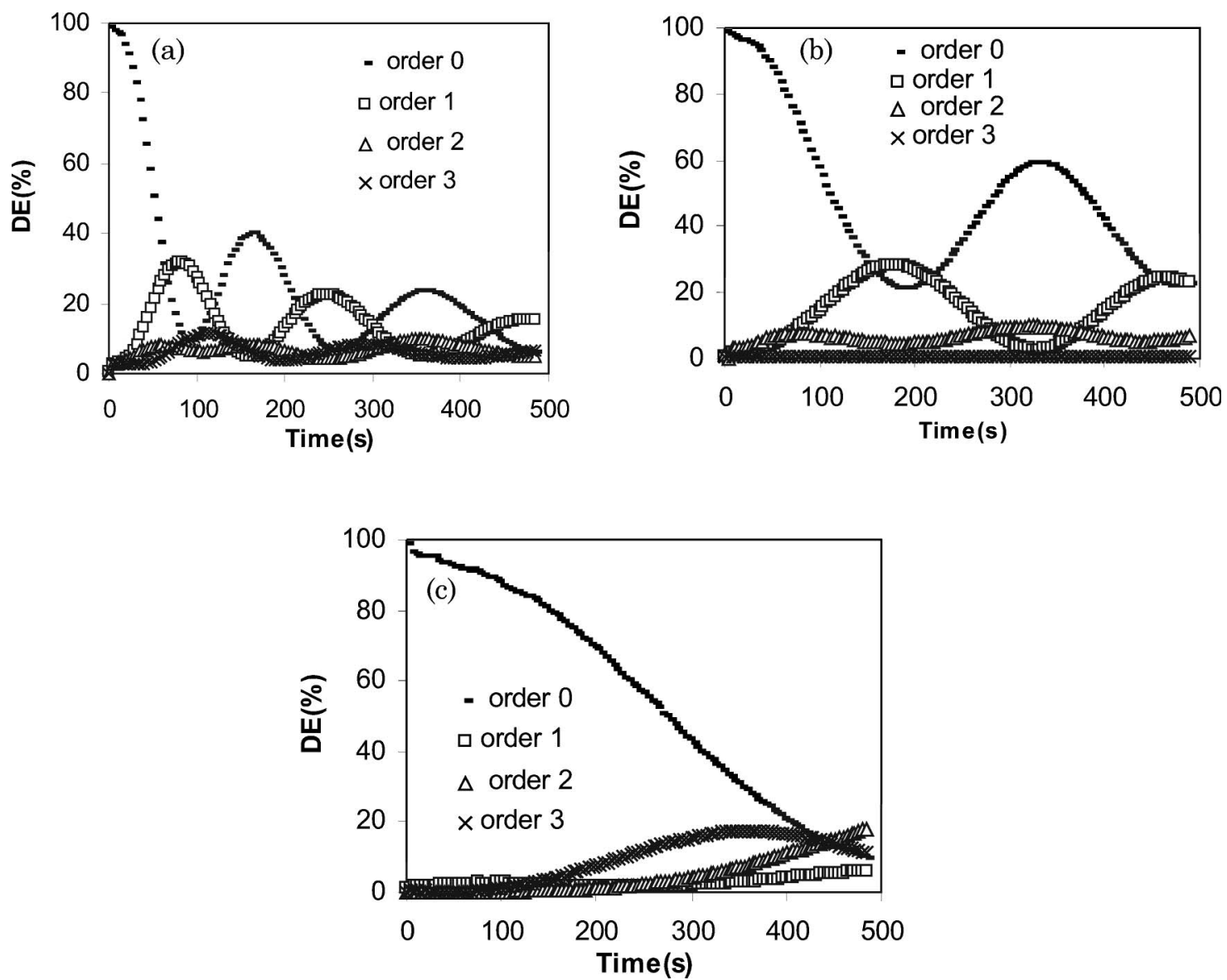

Fig. 5. Diffraction efficiencies of the first four orders for different gratings periods [0.168, 0.336, and $0.672 \mathrm{~mm}$ corespond to (a), (b), and (c), respectively] as a function of the exposition time (105 $\mu \mathrm{m}$ thick, with cross-linker, in transmission).

grating period of $0.168 \mathrm{~mm})$. The results for spatial periods of 0.336 and $0.672 \mathrm{~mm}$ are similar the ones given in the non-cross-linker case; the efficiencies of each order decrease to values lower than $10 \%$ after a few seconds. In the cross-linker case, although results for the two longer spatial periods are similar to the case without a cross-linker, results for the shorter period $(0.168 \mathrm{~mm})$ are clearly different. In a previous work we noted the higher shrinkage when a crosslinker is present at the zero spatial frequency limit [22].

In Subsection 3.C we want to evaluate the profile for the phase modulation. This should give us quantitative information for the kind of periodic profile recorded and the phase-modulation depth. To this goal we have assumed that the periodic profile can be fitted by a Fermi-Dirac function as described in Subsection 2.A.

\section{Profile Shape Estimation}

In this section we analyze the gratings recording in the PVA/AA material and test the capability of this material to store diffractive elements with long periods. To study the phase gratings, we have used a simulated profile based on the Fermi-Dirac function. Usually, in the initial steps of recording $(10 \mathrm{~s})$ only $-1,0$, and +1 diffraction orders can be detected, which implies that we cannot analyze the shape of the profile without making more assumptions. In this sense, there are two important variables that we must take into account in order to characterize the phase-grating profiles recorded in the photopolymers: the depth of the phase shift and the shape of the edges of the grating stored. After a few seconds, more pairs of orders become significant and, so, we can fit the experimental data and obtain a more reliable profile of the phase modulation. Specifically, the procedure used considers nine diffracted orders, from order 0 to 8 . In Table 1 we show the diffraction efficiencies of the first nine transmission orders for a grating with a period of $0.168 \mathrm{~mm}$ (without crosslinker) for different recording times.

In Figs. 9(a) and 9(b), respectively, for the gratings with periods of 0.168 and $0.672 \mathrm{~mm}$, we can see that the phase-modulation profiles initially resemble a rectangle function, which is basically the profile imaged onto the recording plane. In other words, there is a linearity in the material response. On the other hand, the phase depth between dark and bright zones is higher than $2 \pi$, interesting for some diffractive applications. In these figures it is possible to analyze the changes produced by the diffusion processes at the edges of the profile. For longer periods, smoothing and attenuation of the profiles takes place 

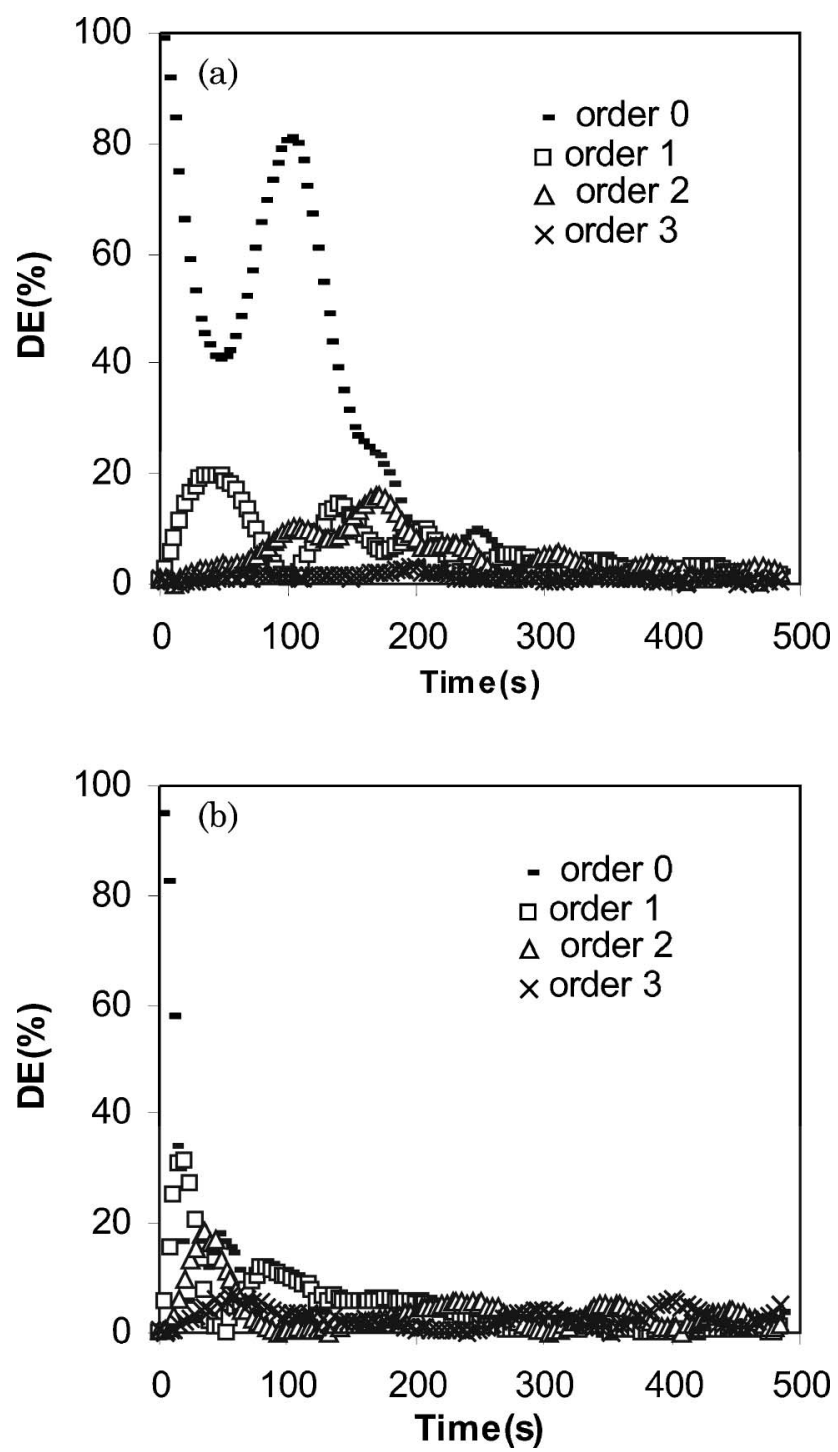

Fig. 6. Diffraction efficiencies of the first four reflected orders for different gratings periods [0.168 and $0.336 \mathrm{~mm}$ correspond to (a) and (b), respectively] as a function of the exposition time (105 $\mu \mathrm{m}$ thick, without cross-linker, in reflection).

after $400 \mathrm{~s}$ of exposure and, for shorter periods, attenuation of the edges can be detected after only $200 \mathrm{~s}$. In principle, in diffractive optics applications, attenuation of the edges produce a degradation of the DOE recorded; in general, this attenuation limits the minimum spot size (resolution) that can be recorded. Furthermore, in binary elements diffraction efficiency is higher for binary profiles. Therefore, the material should basically be used in the range where this attenuation is not so readily apparent. Techniques to fix the phase image, that is, to decrease the diffusion and surface variations after exposition, are required to stop the smoothing of the edges. One possible solution, well tested by O'Neill et al. in PVA/AA-based photopolymers, is the introduction of a sealant [27]. Another important fact we can observe in Fig. $\overline{9(b)}$ is that the phasedepth value of the grating is very similar to the

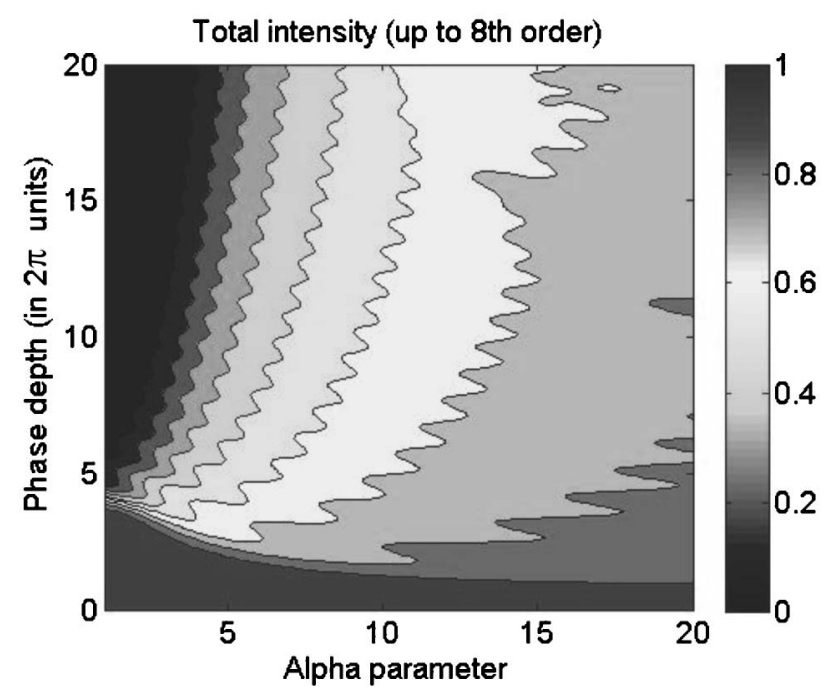

Fig. 7. Sum of diffraction efficiencies of the first eight reflected orders for different values of $\alpha$ and phase depth.

one for the zero spatial frequency limit [21-23]; this is clear evidence of the low importance of diffusion processes for these very long spatial periods for exposures shorter than 500 s. In Fig. 9(c), we show the sensitivity of the fitting procedure using the Fermi-Dirac function. We show the logarithm of the sum of the differences squared for the nine diffracted experimental orders and the numerical values. The $Y$ axis and the $X$ axis show, respectively, the phase depth and the $\alpha$ value. We see how a well-defined global minimum can be obtained.

Similar results are obtained in the analysis of the phase-modulation profiles for compositions with BMA as a cross-linker [Figs. 10(a) and 10(b)]. The fitted profiles evidence the differences in the material behavior for spatial periods of 0.168 [Fig. 10(a)] and $0.672 \mathrm{~mm}$ [Fig. 10(b)]. In addition, in Fig. 10(b), we can see the similarity of the phase depth achieved for very long spatial periods with the phase shift obtained at

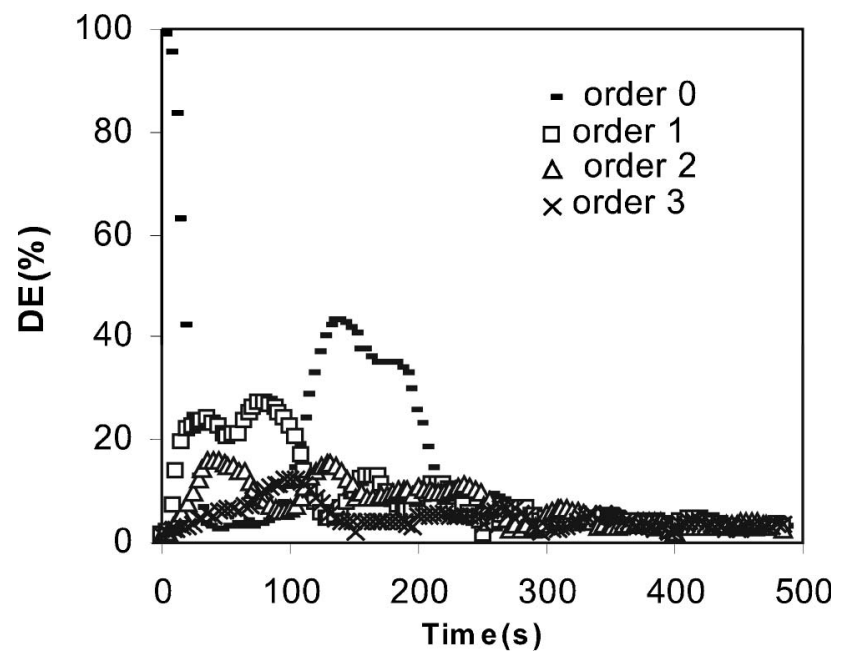

Fig. 8. Diffraction efficiencies of the first four reflected orders for a gratings period of $0.168 \mathrm{~mm}$ as a function of the exposition time (105 $\mu \mathrm{m}$ thick, with cross-linker, in reflection). 
Table 1. Diffraction Efficiencies of the First Nine Orders for a Grating With a Period of $0.168 \mathrm{~mm}$ (Without Cross-Linker)

\begin{tabular}{cccccccccc}
\hline Time $(\mathrm{s})$ & $\mathrm{DE}_{0}(\%)$ & $\mathrm{DE}_{1}(\%)$ & $\mathrm{DE}_{2}(\%)$ & $\mathrm{DE}_{3}(\%)$ & $\mathrm{DE}_{4}(\%)$ & $\mathrm{DE}_{5}(\%)$ & $\mathrm{DE}_{6}(\%)$ & $\mathrm{DE}_{7}(\%)$ & $\mathrm{DE}_{8}(\%)$ \\
\hline 40 & 80.4 & 5.7 & 2.0 & 0.6 & 0.6 & 0.3 & 0.0 & 0.2 & 0.0 \\
80 & 41.2 & 20.3 & 3.7 & 2.0 & 0.7 & 0.4 & 0.1 & 0.2 & 0.1 \\
240 & 35.9 & 2.3 & 5.1 & 4.4 & 7.4 & 3.8 & 2.2 & 0.8 \\
480 & 22.5 & 2.6 & 6.2 & 3.8 & 3.6 & 0.8 & 1.8 & 2.3 & 1.1 \\
\hline
\end{tabular}

the zero spatial frequency limit [22]. The phase depth is constant after $240 \mathrm{~s}$ of exposure and the maximum is around 1.2 times $2 \pi$, as in the case of zero spatial frequency limit.

Next we discuss the profiles obtained for the measurements taken in reflection. At this point in our work, we must take into account that our technique based on measuring the diffraction efficiencies of the different diffraction orders presents some difficulties when applied to the measurements in reflection. As we can see in Figs. 6 and 8, diffraction efficiencies decrease to values lower than $10 \%$ in just $200 \mathrm{~s}$. We discuss in Fig. 7 that these low values were due to the large phase-modulation depths exhibited by the surface profile and, basically, for $\alpha$ values smaller than 5 (i.e., smoothed profiles). These weak values of the first nine orders produce a large uncertainty in the fitting of the profile. In particular, we

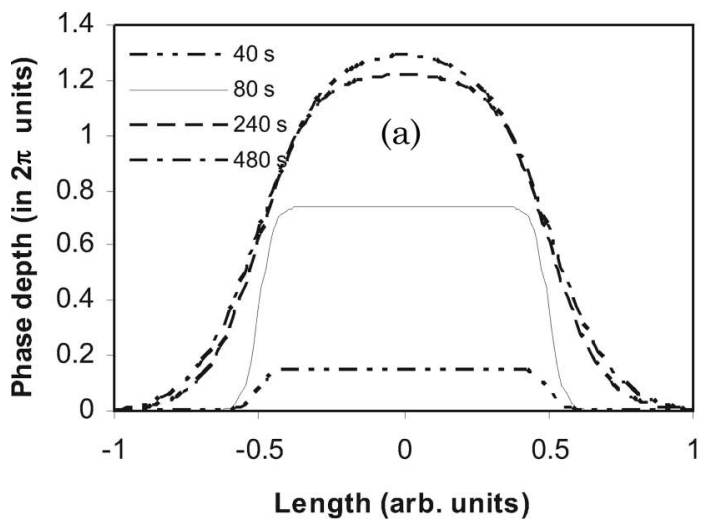

observe that the values obtained for the $\alpha$ parameter look reasonable, providing the expected smoothed profile, but the phase depths $\varphi_{0}$ obtained are even much larger than expected. In fact, we can say that we have an ambiguity in the phase depth because there exists a set of different $\varphi_{0}$ values giving a very similar fit; these values are practically equal modulus $2 \pi$. In the case of the gratings with the larger period $(0.672 \mathrm{~mm})$, this ambiguity in the fitting can be partially solved since we can use additional information. As we have noted Subsection 3.A, for spatial periods of $0.672 \mathrm{~mm}$, diffusion does not play an important role, so we can assume the zero spatial frequency limit behavior and choose the most physically reasonable phase-depth value among the different sets of possibilities modulus $2 \pi$. Using this procedure, in Figs. 11(a) and 11(b), we see the large values of phase depth achieved for the two chemical

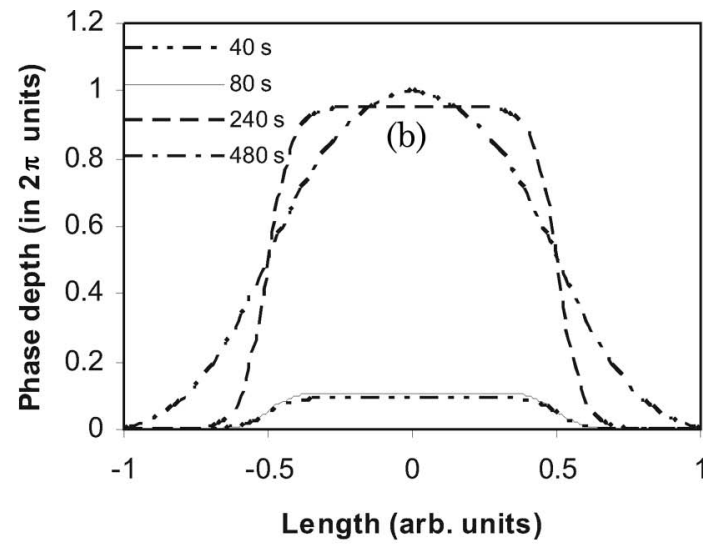

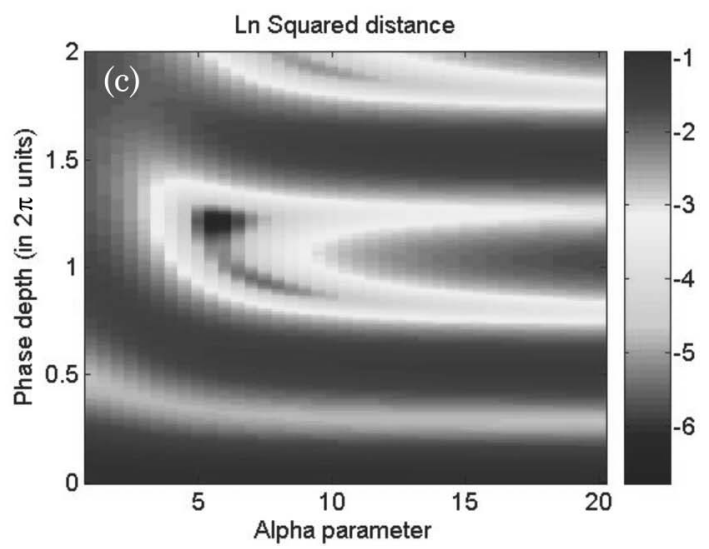

Fig. 9. Fitted phase profiles for different exposure times using Fermi-Dirac distribution ( $105 \mu \mathrm{m}$ thick, without cross-linker, in transmission). (a) Spatial period of $0.168 \mathrm{~mm}$. (b) Spatial period of $0.672 \mathrm{~mm}$. (c) Search for the optimum phase profile after $240 \mathrm{~s}$ of exposure for a layer with a cross-linker. 

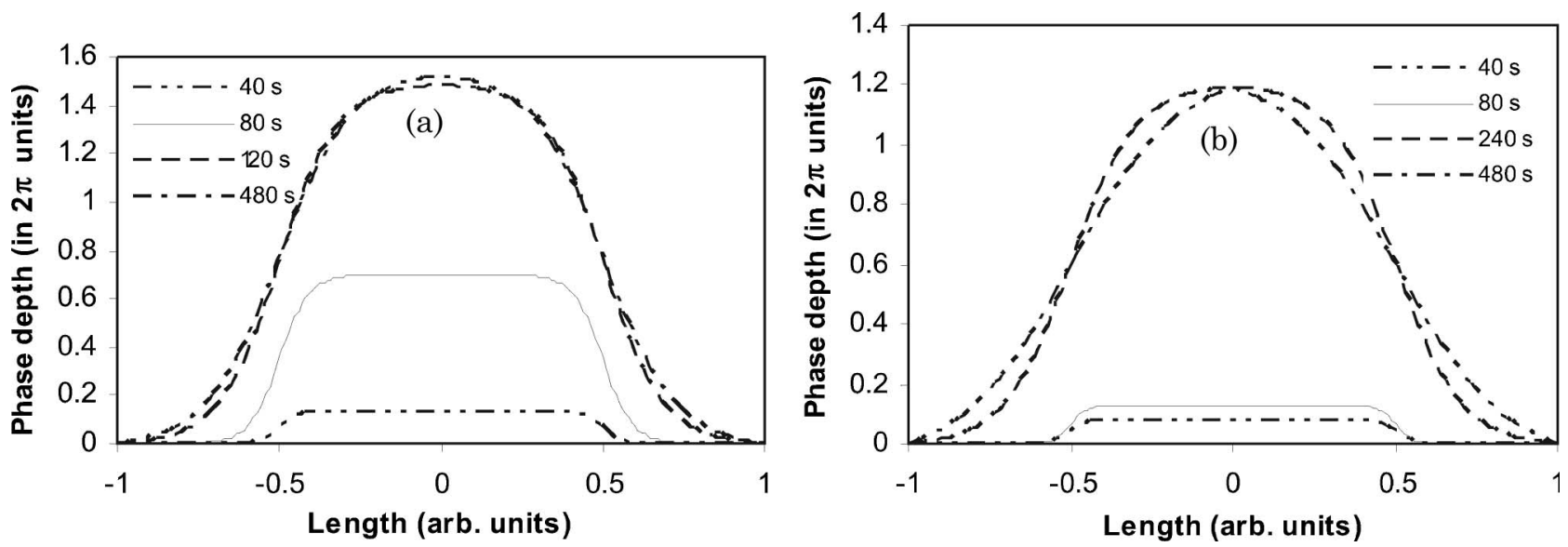

Fig. 10. Fitted phase profiles for different exposure times using Fermi-Dirac distribution (105 $\mu \mathrm{m}$ thick, with cross-linker, in transmission). (a) Spatial period of $0.168 \mathrm{~mm}$. (b) Spatial period of $0.672 \mathrm{~mm}$.

compositions tested and for the gratings with the larger period. Smoothing of the edges as exposure time increases can be clearly observed in the two cases depicted. The validity of the fittings around the phasedepth value considered as physically reasonable is shown in Fig. 11(c), where the local minimum is clearly detected.

At this point, it is interesting to summarize in a table the results obtained in this work. In Table 2 we remark two important aspects of the experimen-

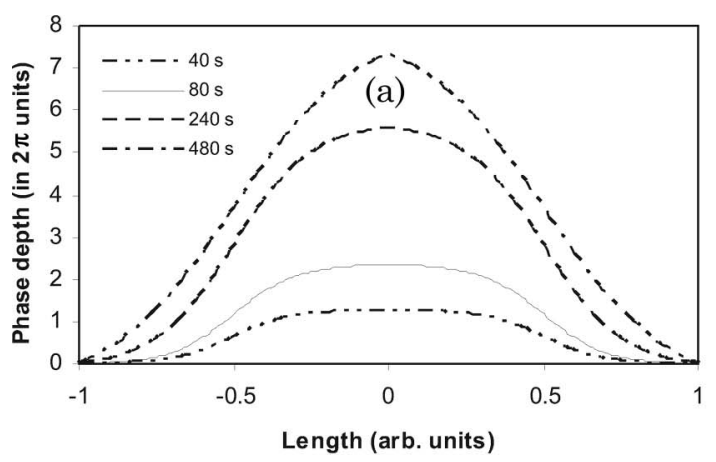

tal results: the maximum value of the diffraction efficiency of the first order $\left(\mathrm{DE}_{1 \max }\right)$ and the highest value of the phase depth obtained using the FermiDirac model $\left(\mathrm{PD}_{\max }\right)$. These values show the material response for each spatial frequency analyzed. It is important to note that we have introduced in Table 2 the values for $\mathrm{PD}_{\max }$ for the spatial period of $0.336 \mathrm{~mm}$ (we do not have depicted the Fermi-Dirac fittings because the interesting spatial periods are the highest and the smallest ones). In addition, we

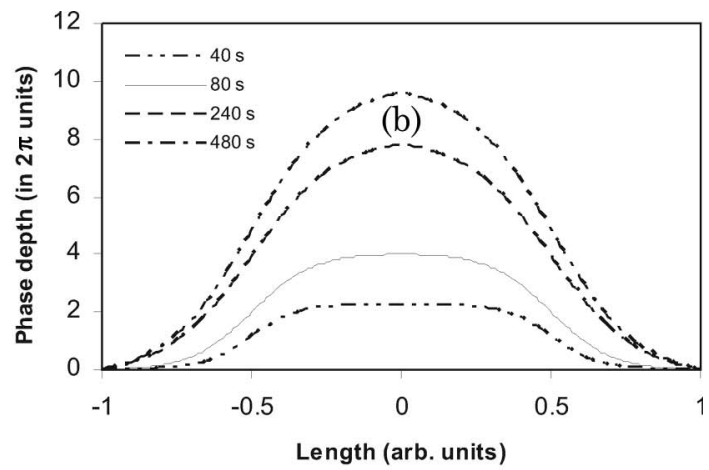

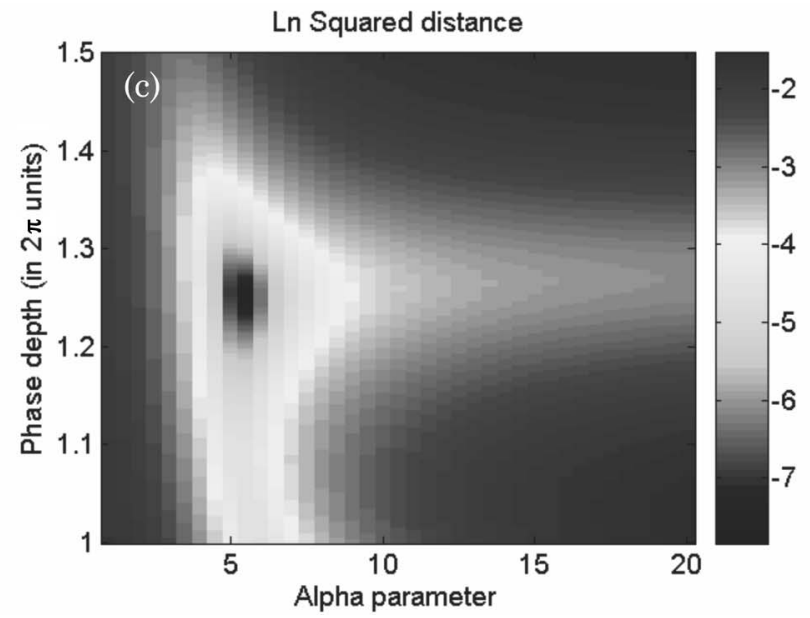

Fig. 11. Fitted surface profiles for different exposure times using Fermi-Dirac distribution (105 $\mu$ m thick with a spatial period of $0.672 \mathrm{~mm}$, in reflection) (a) without cross-linker and (b) with cross-linker. (c) Search for the optimum phase profile after $40 \mathrm{~s}$ of exposure for a layer with a cross-linker. 
Table 2. Summary of the Values of $D E_{1 \max }$ and $\mathrm{PD}_{\max }$ Obtained for Each Spatial Period and Composition

\begin{tabular}{|c|c|c|c|c|c|c|c|c|c|c|c|c|}
\hline \multirow[b]{2}{*}{$\begin{array}{c}\text { Spatial } \\
\text { Period } \\
(\mathrm{mm})\end{array}$} & \multicolumn{6}{|c|}{ Transmission Analysis } & \multicolumn{6}{|c|}{ Reflection Analysis } \\
\hline & $\begin{array}{c}\mathrm{ES} \\
\left(\mathrm{mJ} / \mathrm{cm}^{2}\right)\end{array}$ & $\begin{array}{c}\mathrm{DE}_{1 \max } \\
(\%)\end{array}$ & $\begin{array}{c}\mathrm{PD}_{\max } \\
(2 \pi \text { units })\end{array}$ & $\begin{array}{c}\mathrm{ES} \\
\left(\mathrm{mJ} / \mathrm{cm}^{2}\right)\end{array}$ & $\begin{array}{c}\mathrm{DE}_{1 \max } \\
(\%)\end{array}$ & $\begin{array}{c}\mathrm{PD}_{\max } \\
(2 \pi \text { units })\end{array}$ & $\begin{array}{c}\mathrm{ES} \\
\left(\mathrm{mJ} / \mathrm{cm}^{2}\right)\end{array}$ & $\begin{array}{c}\mathrm{DE}_{1 \max } \\
(\%)\end{array}$ & $\begin{array}{c}\mathrm{PD}_{\max } \\
(2 \pi \text { units })\end{array}$ & $\begin{array}{c}\mathrm{ES} \\
\left(\mathrm{mJ} / \mathrm{cm}^{2}\right)\end{array}$ & $\begin{array}{c}\mathrm{DE}_{1 \max } \\
(\%)\end{array}$ & $\begin{array}{c}\mathrm{PD}_{\max } \\
(2 \pi \text { units })\end{array}$ \\
\hline 0.168 & 55 & 30 & 1.3 & 45 & 32 & 1.5 & 25 & 20 & - & 12.5 & 27 & - \\
\hline 0.336 & 125 & 37 & 1.1 & 90 & 30 & 1.3 & 12.5 & 31 & - & 5 & 29 & - \\
\hline
\end{tabular}

have not introduced the value for $\mathrm{PD}_{\max }$ in the reflection case for spatial periods of 0.168 and $0.372 \mathrm{~mm}$ due to the weak diffraction efficiencies of the first eighth orders, as we have already explained in discussion of Fig. 7. To conclude, we can point out that, in our experiments, we have obtained large values of $\mathrm{DE}_{1 \max }$ (up of $20 \%$ ) and values of $\mathrm{PD}_{\max }$ higher than $2 \pi$ using PVA/AA-based photopolymers. With respect to the energetic sensitivity (ES), the energy necessary to achieve $\mathrm{DE}_{1 \max }$, we note that cross-linker compositions are faster than the ones without. We also note that, for the period $0.672 \mathrm{~mm}$, in transmission the first-order diffraction efficiency evolution does not present a maximum in the exposure time considered, since diffusion is probably acting, but very slowly, because of the large length the monomer has to cross for this long period. When comparing the sensitivity for transmission and reflected orders, we see that sensitivity is clearly larger in the second case, due to the large importance of surface relieve modulations.

\section{Conclusions}

In this work we have tested the feasibility of a PVA/ AA-based photopolymer to record structures with long periods. In particular, we have studied the critical range of spatial frequencies where species diffusion starts to play an important role in the phase-image formation. We have analyzed the different behavior observed at three different spatial frequencies $(5.8,2.9$, and 1.49 lines $/ \mathrm{mm})$. We have studied two different compositions for the photopolymer, with and without a cross-linker. The composition with BMA presents higher energetic sensitivity. In transmission for every different spatial frequency, we achieve phase modulations larger than $2 \pi$ for both compositions. This modulation value makes the material suitable to record diffractive elements. Using a Fermi-Dirac function; we have reported the shape of the profiles and have estimated the phase depth. In this sense, we have shown that, due to diffusion processes, it is not possible to obtain sharp edges with PVA/AA-based materials as exposition time increases. However, at low exposition times, the profiles are rather sharp and provide high diffraction efficiency on the first diffracted orders (except for the $0.672 \mathrm{~mm}$ period), which is interesting for diffractive optics applications. Techniques to fix the phase images, to decrease the diffusion, are required to stop the smoothing of the edges. Furthermore, using measurements in reflection, we have analyzed the variations in the layer surface. In the case of the surface profile, we have obtained that the phase depth of the surface modulation can be very large. In particular, we have demonstrated that the low values of diffracted intensity obtained for the first nine orders are due to the combination of a very large phase depth and a smoothed profile.

This work was supported by the Ministerio de Edicación y Ciencia (Spain) under projects FIS2008-05856-C02-01 and FIS2008-05856-C02-02 and by Generalitat Valenciana under projects GVPRE/2008/137, ACOMP/2009/150, and ACOMP/ $2009 / 160$.

\section{References}

1. J. T. Sheridan and J. R. Lawrence, "Nonlocal-response diffusion model of holographic recording in photopolymer," J. Opt. Soc. Am. A 17, 1108-1114 (2000).

2. Sheridan, M. Downey, and F. T. O'Neill, "Diffusion based model of holographic grating formation in photopolymers: generalized non-local material responses," J. Opt. A Pure Appl. Opt. 3, 477-488 (2001)

3. H. J. Coufal, D. Psaltis, and G. T. Sincerbox, eds., Holographic Data Storage (Springer-Verlag, 2000).

4. W. L. Wilson, K. R. Curtis, K. Anderson, M. C. Tackitt, A. J. Hill, M. Pane, C. Stanhope, T. Earhart, W. Loechel, C. Bergman, K. Wolfgang, C. Shuman, G. Hertrich, K. Parris, K. Malang, B. Riley, and M. Ayer, "Realization of high performance holographic data storage: the inPhase Technologies demonstration platform,” Proc. SPIE 5216, 178-191 (2003).

5. http://www.inphase-technologies.com/news/ 06_june19_RadTech.asp?subn=6_2

6. D. A. Waldman, C. J. Butler, and D. H Raguin, "CROP holographic storage media for optical data storage at greater than $100 \mathrm{bits} / \mu \mathrm{m}^{2}$," Proc. SPIE 5216, 10-25 (2003),

7. Y. Boiko, V. Slovjev, S. Calixto, and D. Lougnot, "Dry photopolymer films for computer-generated infrared radiation focusing elements," Appl. Opt. 33, 787-793 (1994).

8. D. C. O'Shea, T. J. Suleski, A. D. Kathman, and D. W. Prather, Diffractive Optics: Design, Fabrication and Test (SPIE, 2004).

9. T. Sigitas, J. Giedrius, G. Asta, P. Arvydas, O. Vytautas, and A Mindaugas, "Optical characterization of diffractive optical elements replicated in polymers," J. Microlith. Microfab. Microsyst. 5, 807-814 (2006).

10. C. Croutxe-Barghorn and D. Lougnot, "Use of self-processing dry photopolymers for the generation of relief optical elements: a photochemical study," Pure Appl. Opt. 5, 811825 (1996).

11. J. Turunen and F. Wyrowski, Diffractive Optics for Industrial and Commercial Applications (Akademie Verlag, 1997). 
12. P. Cottin, R. A. Lessard, É. J. Knystautas, and Sjoerd Roorda, "Polymer waveguides under ion implantation: optical and chemical aspects," Nucl. Instrum. Methods Phys. Res. B 151, 97-100 (1999).

13. J. Neumann, K. S. Wieking, and D. Kip, "Direct laser writing of surface reliefs in dry, self-developing photopolymer films," Appl. Opt. 38, 5418-5421 (1999).

14. X. T. Li, A. Natansohn, and P. Rochon, "Photoinduced liquid crystal alignment based on a surface relief grating in an assembled cell," Appl. Phys. Lett. 74, 3791-3793 (1999).

15. D. Dantsker, J. Kumar, and S. K. Tripathy, "Optical alignment of liquid crystals,” J. Appl. Phys. 89, 4318-4325 (2001).

16. M. E. Potter, K. Goss, M. A. Neifeld, and R. W. Ziolkowski, "Nanostructure surface relief profiles for high-density optical data storage," Opt. Commun. 253, 56-69 (2005).

17. K. Pavani, I. Naydenova, S. Martin, and V. Toal, "Photoinduced surface relief studies in an acrylamide-based photopolymer," J. Opt. A Pure Appl. Opt. 9, 43-48 (2007).

18. K. Pavani, I. Naydenova, S. Martin, R. Jallapuram, R. G. Howard, and V. Toal, "Electro-optical switching of liquid crystal diffraction gratings by using surface relief effect in the photopolymer," Opt. Commun. 273, 367-369 (2007).

19. C. Neipp, A. Beléndez, S. Gallego, M. Ortuño, I. Pascual, and J. Sheridan, "Angular responses of the first and second diffracted orders in transmission diffraction grating recorded on photopolymer material," Opt. Express 11, 1835-1843 (2003).

20. S. Gallego, M. Ortuño, I. Pascual, C. Neipp, A. Márquez, and A. Beléndez, "Analysis of second and third diffracted orders in volume diffraction gratings recorded on photopolymers," Phys. Scr. T T118, 58-60 (2005).
21. S. Gallego, A. Márquez, D. Méndez, M. Ortuño, C. Neipp, M. L. Alvarez, A. Beléndez, E. Fernández, and I. Pascual, "Real-time interferometric characterization of a PVA based photopolymer at the zero spatial frequency limit," Appl. Opt. 46, 75067512 (2007).

22. S. Gallego, A. Márquez, D. Méndez, M. Ortuño, C. Neipp, M. L. Alvarez, A. Beléndez, E. Fernández, and I. Pascual, "Analysis of PVA/AA based photopolymers at the zero spatial frequency limit using interferometric methods," Appl. Opt. 47, 25572563 (2008).

23. S. Gallego, A. Márquez, D. Méndez, C. Neipp, M. Ortuño, A. Beléndez, E. Fernández, and I. Pascual, "Direct analysis of monomer diffusion times in polyvinyl/acrylamide materials," Appl. Phys. Lett. 92, 073306 (2008).

24. M. V. Kessels, M. E. Bouz, R. Pagan, and K. Heggarty, "Versatile stepper based maskless microlithography using a liquid crystal display for direct write of binary and multilevel microstructures," J. Micro/Nanolith. MEMS MOEMS 6, 033002 (2007).

25. A. Márquez, C. Iemmi, I. Moreno, J. A. Davis, J. Campos and M. J. Yzuel, "Quantitative prediction of the modulation behavior of twisted nematic liquid crystal displays," Opt. Eng. 40, 2558-2564 (2001).

26. A. Márquez, J. Campos, M. J. Yzuel, I. Pascual, A. Fimia, and A. Beléndez, "Production of computer-generated phase holograms using graphic devices: application to correlation filters," Opt. Eng. 39, 1612-1619 (2000).

27. F. T. O'Neill, J. R. Lawrence, and J. T. Sheridan, "Improvement of holographic recording material using aerosol sealant," J. Opt. A Pure Appl. Opt. 3, 20-25 (2001) 